\title{
Correction : Les schémas de subdivision de Besicovitch et de Cantor
}

\author{
Serge Dubuc ${ }^{1}$ (D)
}

(c) Fondation Carl-Herz and Springer Nature Switzerland AG 2022

\section{Correction to: Ann. Math. Québec https://doi.org/10.1007/s40316-020-00131-9}

\section{Correction du théorème 1}

Théorème 1 Soit un schéma de subdivision de Besicovitch $L_{0}, L_{1}, L_{2}, \ldots$ dont la limite est $f$, si $\delta_{n}=O\left(1 / 2^{n}\right)$ où $\delta_{n}$ est le diamètre de la partition engendrée par les abscisses de $L_{n}$, alors aucune dérivée à droite finie de $f$ n'existe. Il en est de même pour les dérivées à gauche.

Démonstration Quitte à changer la position de l'origine et les échelles des axes, on peut supposer que $L_{0}$ a pour extrémités les points $(0,0)$ et $(1,1)$. Nous raisonnons par l'absurde en supposant qu'il existe un nombre $\xi \in[0,1)$ tel que la dérivée à droite $D=f_{+}^{\prime}(\xi)$ existe comme limite finie. Nous posons $M=\sup _{n}\left(2^{n} \delta_{n}\right)$. Soit $\varepsilon>0$ un nombre que nous choisirons tantôt. Soit $n$ un entier suffisamment grand, on détermine les abscisses consécutives de $L_{n+1}$, $a_{n}<c_{n}<d_{n}<e_{n}$ où $a_{n}$ est l'abscisse de $L_{n}$ à la droite de $\xi$ qui lui soit le plus proche et que $c_{n}, d_{n}, e_{n}$ sont les 3 voisins immédiats à droite de $a_{n}$ parmi les abcisses de $L_{n+1}$. On exige de $n$ que

$$
|f(x)-f(\xi)-D(x-\xi)|<\varepsilon(x-\xi) \text { lorsque } x \text { est remplacé par } c_{n}, d_{n}, e_{n} .
$$

Dans un premier temps, nous supposons que $D \leq 0$. Puisque par construction, $f\left(c_{n}\right)=$ $f\left(e_{n}\right)$ et $f\left(d_{n}\right)=f\left(c_{n}\right) \pm 1 / 2^{n+1}$, parmi les trois abscisses $c_{n}, d_{n}, e_{n}$, nous pouvons en choisir deux $x_{1}<x_{2}$ tels que $f\left(x_{2}\right)=f\left(x_{1}\right)+1 / 2^{n+1}$. Sachant que $x_{1}-\xi<x_{2}-\xi<2 M / 2^{n}$, on obtient que $f\left(x_{2}\right)-f(\xi)-D\left(x_{2}-\xi\right)<2 M \varepsilon / 2^{n}$ et $f\left(x_{1}\right)-f(\xi)-D\left(x_{1}-\xi\right)>-2 M \varepsilon / 2^{n}$. Par soustraction, on obtient $1 / 2^{n+1}-D\left(x_{2}-x_{1}\right)<4 M \varepsilon / 2^{n}$. C'est une impossible inégalité si l'on choisit $\varepsilon=1 /(8 M)$.

Si $D>0$, parmi les trois abscisses $c_{n}, d_{n}, e_{n}$, nous en choisissons deux $x_{1}<x_{2}$ tel que $f\left(x_{2}\right)=f\left(x_{1}\right)-1 / 2^{n+1}$. En transposant les arguments de tantôt, on obtient que $f\left(x_{2}\right)-$

The original article can be found online at https://doi.org/10.1007/s40316-020-00131-9.

\section{Serge Dubuc}

serge.dubuc@umontreal.ca

1 Département de mathématiques et de statistique, Université de Montréal, C.P. 6128, Succ. Centre-ville, Montréal, QC H3C 3J7, Canada 
$f(\xi)-D\left(x_{2}-\xi\right)>-2 M \varepsilon / 2^{n}$ et $f\left(x_{1}\right)-f(\xi)-D\left(x_{1}-\xi\right)<2 M \varepsilon / 2^{n}$. Par soustraction, on obtient $-1 / 2^{n+1}-D\left(x_{2}-x_{1}\right)>-1 / 2^{n+1}$, une impossible inégalité.

Pour ce qui est de l'inexistence de dérivée à gauche finie, on procède par symétrie. Soit $L_{n}^{\prime}$ l'image de $L_{n}$ sous la symétrie centrale $(x, y) \rightarrow(1-x, 1-y)$, alors la suite $L_{n}^{\prime}$ converge vers la fonction $x \rightarrow 1-f(1-x)$. Cette fonction n'admet aucune dérivée à droite finie et $f$ elle-même n'admet aucune dérivée à gauche finie.

\section{La fonction de Besicovitch et sa variante}

La tradition reconnaît que la fonction de Besicovitch provient de Singh [5] : si $S:[0,1] \rightarrow$ $\mathbb{R}$ est la fonction de Singh, alors la fonction de Besicovitch, définie sur [0,2], est $y=$ $f(x)=S(x / 2)$. La fonction de Besicovitch peut être construite à l'aide d'une suite de lignes polygonales $L_{0}, L_{1}, L_{2}, \ldots$ dont chaque côté est muni d'un indice $m$ de partage et d'un signe $s= \pm 1$ d'orientation. La ligne de départ $L_{0}$ est $\{(0, f(0)=0),(1, f(1)=1),(2, f(2)=$ $0)\}$ et chacun des deux côtés est d'indice $m=1$ et de signe $s=-1$. À chaque étape, un côté $A(a, f(a)), B(b, f(b))$ d'indice $m$ et de signe $s$ est subdivisé en 4 côtés donnant lieu à une ligne $A C D E B$. Les sommets intercalés sont dans l'ordre $(c, f(c)),(d, f(d))$, $\left(e, f(e)\right.$ où $d=(a+b) / 2, c=d-(b-a) / 2^{2 m+1}, e=d+(b-a) / 2^{2 m+1}$ alors que $f(c)=f(e)=(f(a)+f(b)) / 2$ et $f(d)=(f(a)+f(b)) / 2+s(f(b)-f(a))$. Les indices associés à ces nouveaux côtés sont dans l'ordre $m+1,1,1, m+1$ tandis que les signes associés sont $s,-s,-s, s$. La fonction $f$ est d'abord définie sur un ensemble dense dans $[0,2]$, puis se prolonge par continuité à tout $[0,2]$.

On peut modifier le schéma de Besicovitch précédent en décidant que le signe d'orientation associé à chaque côté est toujours négatif. On désignera par $y=B(x)$ la fonction limite de ce second schéma. Dubuc a cru que la fonction $B$ était la fonction de Besicovitch, ce qui ne correspond pas à l'usage. Pour éviter toute confusion, nous proposons de dire que $B$ est la variante de la fonction de Besicovitch. Partant de là, il faut corriger l'énoncé du théorème 9 et nous retirons dans le texte [3] le paragraphe consécutif à ce théorème qui critiquait un résultat central de Besicovitch.

Théorème 9 La dérivée à droite de $y=B(x)$, la variante de la fonction de Besicovitch, en $4 / 7$ vaut $+\infty$.

\section{Correction du théorème 11}

Théorème 11 Il est impossible que la fonction de Besicovitch, $y=f(x)$, ait une dérivée à droite égale à $\pm \infty$ en un endroit donné. Il en est de même pour la dérivée à gauche.

Démonstration Soit $L_{0}, L_{1}, L_{2}, \ldots$ la suite de lignes polygonales dans la construction de la fonction de Besicovitch $y=f(x)$. Soit $x \in[0,2)$ un endroit donné. Au nombre $x$ correspond une suite de chiffres $c_{0}, c_{1}, c_{2}, \ldots$ de la manière suivante: si $n \geq 0$ et si $[(a, f(a)),(b, f(b))]$ est le kième côté de $L_{n}$ tel que $a \leq x<b$, alors $c_{n}$ est le reste de la division de $k-1$ par 4. On dira que la suite $c_{n}$ est l'adresse de $x$. On désignera par $s_{n}$ le signe associé du kième côté et $\Delta_{n}=f(b)-f(a)= \pm 1 / 2^{n}$ l'accroissement des ordonnées de ce côté. Partant de là, nous distinguons 4 cas.

Cas 1 : il y a une infinité de 1 dans l'adresse de $x$. Soit un entier $n$ pour lequel $c_{n}=1$, on désigne par $[(a, f(a)),(b, f(b))]$ le côté de $L_{n}$ tel que $a \leq x<b$. Ce côté est remplacé par 
a) $f(b)>f(a), s=+1$

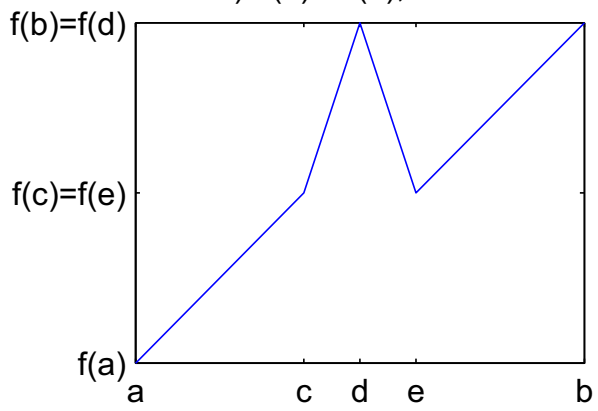

c) $f(b)<f(a), s=+1$

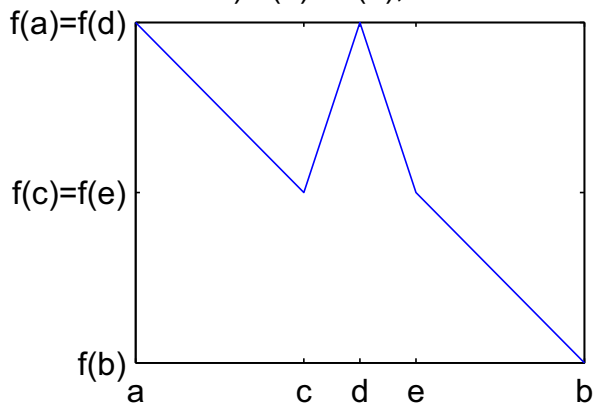

b) $f(b)>f(a), s=-1$

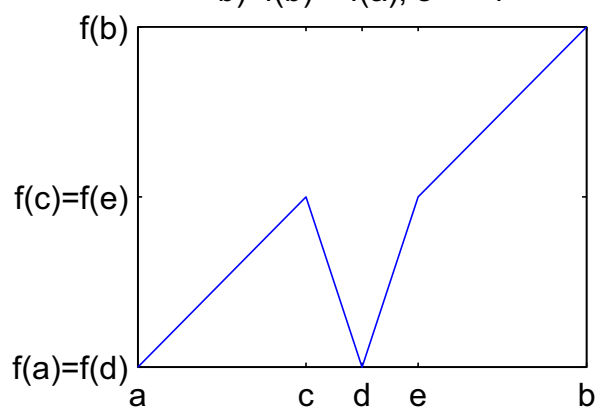

d) $f(b)<f(a), s=-1$

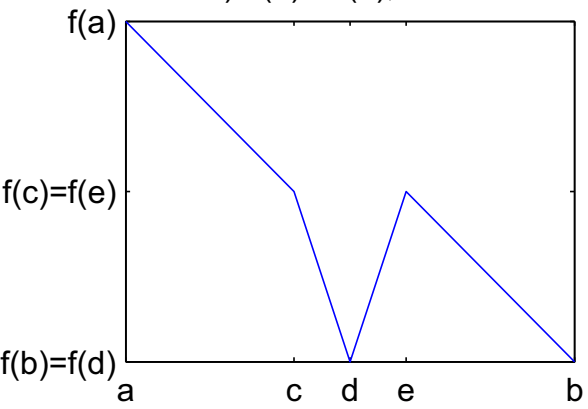

Fig. 1 Quatre types de subdivision pour une subdivision de Besicovitch

la ligne polygonale de 5 sommets $(a, f(a)),(c, f(c)),(d, f(d)),(e, f(e)),(b, f(b))$. Il y a quatre possibilités illustrées par la Fig. 1. Chaque fois, $f(e)=f(c)$ et $c \leq x<d$. Il existe donc un nombre $x^{\prime} \in(d, e]$ tel que $f\left(x^{\prime}\right)=f(x)$. En $x$, il y a une accumulation de quotients différentiels nuls, $f\left[x, x^{\prime}\right]=0$. La dérivée à droite au point $x$ ne peut pas égaler $\pm \infty$.

Cas 2 : il y a une infinité de 2 dans l'adresse de $x$, mais seulement un nombre fini de 1. Soit un entier $N$ tel que $c_{n} \neq 1$ tout $n>N$. Nous considérons les deux suites $\Delta_{n}$ et $s_{n}$ pour $n>N$. Un examen des 4 subdivisions de la Fig. 1 montre la propriété suivante : si $c_{n}=0$ ou 3 , alors $s_{n} \Delta_{n}$ et $s_{n+1} \Delta_{n+1}$ sont de même signe tandis que $s_{n} \Delta_{n}$ et $s_{n+1} \Delta_{n+1}$ ont des signes opposés lorsque $c_{n}=2$. De là, on obtient qu'il existe un entier $n$ tel que $c_{n}=2$ et le signe de $\Delta_{n}$ est $s_{n}$. Dans la même figure, en observant les subdivisions a) et d), on voit que $d \leq x<e$, $f(d)=f(b)$. Il existe donc un nombre $x^{\prime} \in(e, b]$ tel que $f\left(x^{\prime}\right)=f(x)$. Comme tantôt, en $x$, il y a une accumulation de quotients différentiels nuls. La dérivée à droite au point $x$ ne peut pas égaler $\pm \infty$.

Cas 3 : les chiffres 1 et 2 n'apparaissent qu'un nombre fini de fois dans l'adresse de $x$ alors que le chiffre 0 apparaît une infinité de fois. On suppose que $N$ est le plus petit entier tel que pour tout $n>N, c_{n} \in\{0,3\}$. Désignons par $C_{n}=\left[\left(a_{n}, f\left(a_{n}\right),\left(b_{n}, f\left(b_{n}\right)\right]\right.\right.$ le côté de $L_{n}$ tel que $a_{n} \leq x<b_{n}$. L'indice de partage de $C_{N}$ est 1 et de là, pour tout $n>N$, l'indice de partage de $C_{n}$ est $n-N+1$. On remarque que $b_{N+1}-a_{N+1}=(1-1 / 4)\left(b_{N}-a_{N}\right) / 2$, $b_{N+2}-a_{N+2}=\left(1-1 / 4^{2}\right)\left(b_{N+1}-a_{N+1}\right) / 2, b_{N+3}-a_{N+3}=\left(1-1 / 4^{3}\right)\left(b_{N+2}-a_{N+2}\right) / 2, \ldots$ Ce qui entraîne que $b_{n}-a_{n}=\left(b_{N}-a_{N}\right) 2^{N-n} \prod_{m=1}^{n-N}\left(1-1 / 4^{m}\right)$.

Soit $n>N$ tel que $c_{n}=0$, le quotient différentiel $f\left(b_{n}\right)-f(x) /\left(b_{n}-x\right)$ est majoré en valeur absolue par $2 /\left(2^{N}\left(b_{N}-a_{N}\right) \prod_{m=1}^{\infty}\left(1-1 / 4^{m}\right)\right)$ puisque $\left|f\left(b_{n}\right)-f(x)\right| \leq 1 / 2^{n}$ et 
$b_{n}-x>\left(b_{n}-a_{n}\right) / 2$. Comme le chiffre 0 apparaît une infinité de fois dans l'adresse de $x$, il existe une accumulation de quotients différentiels $f\left[x, b_{n}\right]$ bornés par une même quantité. La dérivée à droite au point $x$ ne peut pas égaler $\pm \infty$.

Cas 4 : les chiffres 0,1 et 2 n'apparaissent qu' un nombre fini de fois dans l'adresse de $x$. On choisit un entier $N$ tel que pour tout $n \geq N, c_{n}=3$. Désignons encore par $\left(a_{n}, f\left(a_{n}\right)\right.$, ( $b_{n}, f\left(b_{n}\right)$ les deux sommets consécutifs de $L_{n}$ tels que $a_{n} \leq x<b_{n}$. Le fait que $c_{N}=3$ entraîne que $b_{N+1}=b_{N}$; de même, $b_{N+2}=b_{N}$ et ainsi de suite, pour tout $n>N, b_{n}=b_{N}$. D'autre part, pour tout $n, b_{n}-a_{n}<1 / 2^{n}$. De là, l'inégalité, $b_{N}-x<1 / 2^{n}$. Lorsque $n$ est suffisamment grand, cette dernière inégalité est impossible. Le cas 4 ne se produit jamais.

Enfin la symétrie de $f$ par rapport à la verticale $x=1$ donne lieu à l'équation $f(x)=$ $f(2-x)$ et implique que la dérivée à gauche n'est jamais égale à $\pm \infty$.

Les théorème 1 et 11 donnent une démonstration simple du célèbre résultat de Besicovitch que sa fonction n'admet en aucun endroit une dérivée unilatérale, ni finie, ni infinie. Nous indiquons quatre endroits où ce résultat est établi : chez les auteurs Besicovitch (pp. 548-556) [1], Singh (pp. 61-69) [5], Jeffery (pp. 172-181) [4] et Ciesielki [2]. Notre démonstration et celle de Ciesielski sont les plus courtes.

Nous remercions le professeur Ciesielski d'avoir aimablement attiré notre attention sur les arguments inadéquats dans la démonstrationa initiale des théorèmes 1 et 11 .

\section{References}

1. Besicovitch A. S.: Étude des fonctions continues dans le cadre de la question de leur dérivabilité. (en russe) Mat. Sb. 31, (1924) 529-556.

2. Ciesielski, K. C.: Continuous maps admitting no tangent line: A centennial of Besicovitch functions. Amer. Math. Montly (accepted in 2021).

3. Dubuc, S.: Les schémas de subdivision de Besicovitch et de Cantor. Ann. Math. Que. 44, 329-347 (2020).

4. Jeffery, R. L.: The theory of functions of a real variable. Reprint of the 1953 original. Dover Publications, New York, 1985

5. Singh, A. N.: On functions without one-sided derivatives. Proc. Benares Math. Soc. (N.S.) 3, 55-69 (1941)

Publisher's Note Springer Nature remains neutral with regard to jurisdictional claims in published maps and institutional affiliations. 